\title{
Análisis palinológico de mieles comerciales monoflorales
}

\author{
David Rodríguez de la Cruz, Estefanía Sánchez Reyes, Silvia Sánchez Durán y José Sánchez Sánchez
}

Resumen: Rodríguez de la Cruz, D.; Sánchez Reyes, E.; Sánchez Durán, S. \& Sánchez Sánchez, J. 2013. Análisis palinológico de mieles comerciales monoflorales. Bot. Complut. 37: 171-180.

Se estudiaron 28 muestras de mieles etiquetadas como monoflorales de diverso tipo y de origen español: azahar (11), eucalipto (10) y romero (7), a través de los pertinentes métodos cuantitativos y cualitativos. El análisis cuantitativo mostró un contenido medio de elementos botánicos mayor en las mieles de eucalipto (354497 por 10 gramos de miel) que en las mieles de azahar y romero (100111 y 76705, respectivamente). El estudio cualitativo de las mieles de romero y azahar reflejó valores de riqueza polínica semejantes (media de 38 y 41 formas polínicas, respectivamente), más bajos para las mieles de eucalipto (34), propias todas de ellas de la flora peninsular, salvo en dos muestras, una de miel de azahar y otra de eucalipto, con tipos polínicos propios de flora sudamericana. Asimismo, las mieles de romero superaron el 10\% de representación para el tipo Salvia verbenaca, mientras que en una y dos muestras de mieles de azahar y eucalipto no alcanzaron los umbrales de $10 \%$ y $70 \%$ de los tipos Citrus sinensis y Myrtus communis, respectivamente.

Palabras clave: polen, miel, origen, control de calidad.

Abstract: Rodríguez de la Cruz, D.; Sánchez Reyes, E.; Sánchez Durán, S. \& Sánchez Sánchez, J. 2013. Palynological analysis of unifloral commercial honeys. Bot. Complut. 37: 171-180.

Twenty eight honey samples labelled as monofloral and Spanish were studied by means of quantitative and qualitative methods: orange blossom (11), eucalyptus (10) and rosemary (7). Quantitative analysis displayed a higher number of botanical elements in eucalyptus honeys ( $354497 / 10 \mathrm{~g}$ of honey) than in orange blossom and rosemary honeys (100111 y 76705, respectively). Qualitative study showed high pollen richness in rosemary and orange blossom honyes (an average of 38 and 41 types of pollen, respectively), lower in the case of eucalyptus honeys (34). All of these types of pollen belonged to Iberian species, except in two samples (orange blossom and eucalyptus) with pollen grains from South America flora. Rosemary honeys reached $10 \%$ of Salvia verbenaca type, whereas one and two samples of orange blossom and eucalyptus did not exceed $10 \%$ and $70 \%$ of Citrus sinensis y Myrtus communis types, respectively.

Key words: pollen, honey, origin, quality control.

\section{INTRODUCCIÓN}

El aumento progresivo en el consumo de productos naturales ha motivado durante los últimos años una creciente necesidad en el desarrollo de métodos que permitan evaluar y analizar su calidad. En el caso de la miel, un control estricto de su calidad no sólo se fundamenta en el análisis de sus caracteres físico-químicos, microbiológicos o sensoriales, sino también en análisis polínicos que permiten determinar su origen botánico y geográfico y evitan el fraude comercial (Jones \& Bryant 1996). La normativa actual que regula el mercado europeo de la miel (Directiva 2001/110/CE del Consejo de 20 de diciembre de 2001 relativa a la miel) considera, entre otros aspectos, que este producto puede verse etiquetado con indicaciones que hagan referencia a su origen vegetal y geográfico, siempre y cuando proceda del origen indicado, dife- renciando además dos tipos de miel según su origen vegetal, mieles de flores o néctar y mieles de mielada. La falta de concreción en las normativas actuales para delimitar el origen botánico y geográfico de las mieles de néctar a través de criterios palinológicos ha llevado a considerar en las últimas décadas, una miel como monofloral cuando el porcentaje de representación de un tipo polínico es superior al 45\%. Algunos granos de polen, como los de Citrus L., Tilia L. o Lavandula L., se encuentran hiporrepresentados en los espectros polínicos, mientras que otros como Eucalyptus L'Hér., Castanea sativa Mill. están hiperrepresentados, dando lugar a mieles que son consideradas como monoflorales cuando ese tipo polínico alcanza, al menos, el 90\% (Maurizio 1979).

Esta variedad ha conllevado a su vez un buen número de trabajos que profundizaban en el estudio polínico de mieles monoflorales ya conocidas, sobretodo en localiza-

\footnotetext{
${ }^{1}$ Centro Hispano-Luso de Investigaciones Agrarias (CIALE), Universidad de Salamanca. C/Río Duero 12, 37185 Villamayor (Salamanca). droc@usal.es, fani_sanchez@usal.es, sylvias8@usal.es,jss@usal.es

Recibido: 20 diciembre 2012. Aceptado: 8 enero 2013.
} 
ciones geográficas puntuales de diverso interés (Terrab et al. 2004a; De Sá-Otero et al. 2006) o incluso estudios sobre mieles monoflorales poco conocidas (Terrab et al. 2004b). Sin embargo, existen pocos artículos que analicen el espectro polínico de mieles monoflorales comerciales (Sánchez Sánchez et al. 1996, Seijo \& Aira 1999, de Sá Otero et al. 2011), motivo por el cual aportamos datos que redunden en un mayor conocimiento de las mismas y que permitan decidir si se encuentran dentro de los criterios de origen botánico y geográfico que deben cumplir.

\section{MATERIALES Y MÉTODOS}

Se analizaron 28 muestras de mieles comerciales etiquetadas como monoflorales y de origen español, once de ellas denominadas como azahar, diez como eucalipto y siete como romero. Todas ellas fueron adquiridas en el período 2002-2008 y a partir de distintos lotes de la misma marca comercial, según se indica en la tabla 1.

Para el análisis cuantitativo a través del microscopio óptico se utilizaron $50 \mathrm{~g}$ de miel al natural siguiendo la metodología de Loveaux et al. (1978), mientras que para el análisis cualitativo se utilizó el método acetolítico de Erdtman (1960) y las modificaciones propuestas por Hideux (1972).

La identificación de las distintas formas polínicas se realizó gracias a diferentes claves sobre morfología polínica (Valdés et al. 1987, Carretero 1989) y a la palinoteca del Departamento de Botánica de la Universidad de Salamanca, junto a otros atlas polínicos para aquellos tipos de polen ajenos a la flora peninsular (Heusser 1971, Markgraf \& D'Antoni 1978). La filiación de cada forma polínica con un tipo polínico se llevó a cabo siempre que fue posible, puesto que en algunos casos sólo se

\section{Azahar}

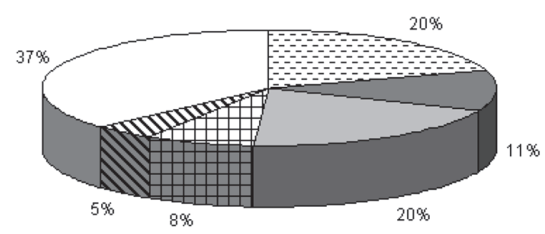

Romero

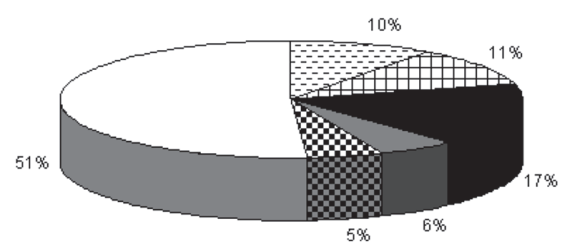

pudo llegar a nivel genérico (Acer) 0 a otros niveles (otras Cistaceae, Liguliflorae).

\section{RESULTADOS}

Los resultados del análisis cuantitativo indicaron que las muestras poseen un contenido medio de elementos botánicos atendiendo a las clases definidas por Maurizio (Loveaux et al. 1978), pero variable en número en función del tipo de miel analizada (Tabla 1). En el caso de las mieles de azahar y romero, el número de elementos botánicos (NEBT) medio en $10 \mathrm{~g}$ de miel para las muestras analizadas fue de 100111 y 76705 , respectivamente, alcanzando valores máximos de 151429 en A10 y 111429 en R6, y mínimos de 55000 en A1 y 58000 en R3. Atendiendo a la clasificación de Maurizio (1939), siete muestras de mieles de azahar y seis de romero se incluyeron en la Clase II, mientras que cuatro de azahar y una única miel de romero se adscribieron a la Clase III. Por el contrario, las muestras etiquetadas como "miel de eucalipto" presentaron un NEBT medio de 354497, con 556000 en E9 como valor más elevado y 248100 en E3 como valor mínimo. Salvo una muestra incluida en la Clase IV, todas las muestras de miel de eucalipto se catalogaron dentro de la Clase III. Los elementos característicos de mielada (ECM) fueron nulos o muy bajos en todas las muestras comerciales analizadas, exceptuando dos muestras de miel de azahar (A3 y A4) y una muestra de miel de eucalipto (E3), que presentaron valores de 18947,12083 y 20000, respectivamente, con un índice de mielada (ECM/Polen Total) muy bajo $(0,15 ; 0,10$ y 0,08$)$.

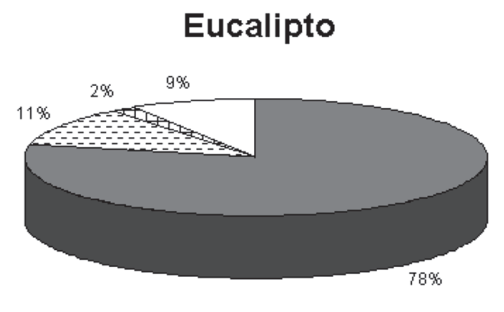

$$
\begin{aligned}
& \square \text { Echium plantagineum } \\
& \square \text { Mytus communis } \\
& \square \text { Citrus sinensis } \\
& \text { ⿴囗ytisus scoparius } \\
& \square \text { Crataegus monogyna } \\
& \square \text { Salvia verbenaca } \\
& \square \text { Lavandula stoechas } \\
& \square \text { Otros }
\end{aligned}
$$

Fig. 1- Representación media de los tipos polínicos más abundantes en las muestras de miel analizadas. Se han tenido en cuenta las formas polínicas adscritas a especies que aportan néctar. 
Respecto al análisis cualitativo de todas las muestras estudiadas, y siguiendo los criterios marcados por Pérez de Zabalza (1989), debe resaltarse que todas ellas mostraron una riqueza polínica alta pues se identificaron más de 20 tipos polínicos, pertenecientes, al menos, a 18 familias botánicas. Según se indica en la Tabla 1, las muestras pertenecientes a mieles etiquetadas como "romero" tienen valores de riqueza polínica más elevados, con respecto a las inscritas como "azahar" y "eucalipto", con valores más bajos. El resto de resultados obtenidos a través del análisis cualitativo serán expuestos para cada tipo de miel analizada por separado, pudiéndose visualizar de forma resumida en la Tabla 2 con el espectro polínico completo en cada una de ellas a través de los porcentajes medios de representación así como si se consideran sólo las formas polínicas nectaríferas.

En las once muestras de miel de azahar se identificaron 44 formas polínicas correspondientes a 27 familias, presentando una media de 27 tipos polínicos concernientes a 19 familias, y siendo A11 y A3 la muestras con mayor y menor riqueza polínica, respectivamente. La familia Leguminosae es la que mostró mayor diversidad de formas polínicas, con cinco, seguida por Compositae y Rosaceae con cuatro, y Labiatae con tres. Asimismo, todas estas familias, junto con Cistaceae, Cruciferae, Liliaceae, Myrtaceae, Oleaceae, Plantaginaceae y Rutaceae,

Tabla 1

Datos de las muestras estudiadas. NEBT: número de elementos botánicos.

\begin{tabular}{|c|c|c|c|c|c|c|}
\hline Muestra & $\begin{array}{l}\text { Denominación } \\
\text { Comercial }\end{array}$ & $\begin{array}{l}\text { NEBT por } 10 \\
\text { g de miel }\end{array}$ & $\begin{array}{l}\text { Clase de } \\
\text { Maurizio }\end{array}$ & $\begin{array}{l}\mathrm{N}^{0} \text { tipos } \\
\text { polínicos }\end{array}$ & $\begin{array}{c}\mathrm{N}^{0} \text { tipos } \\
\text { polínicos } \\
\text { (nectaríferos) }\end{array}$ & $\mathrm{N}^{0}$ familias \\
\hline A1 & Azahar & 55000 & II & 28 & 22 & 21 \\
\hline A2 & Azahar & 81000 & II & 27 & 21 & 19 \\
\hline A3 & Azahar & 144736 & III & 24 & 18 & 18 \\
\hline A4 & Azahar & 129167 & III & 25 & 19 & 18 \\
\hline A5 & Azahar & 91500 & II & 28 & 23 & 20 \\
\hline A6 & Azahar & 90500 & II & 27 & 21 & 20 \\
\hline A7 & Azahar & 84246 & II & 26 & 22 & 18 \\
\hline A8 & Azahar & 76087 & II & 25 & 19 & 18 \\
\hline A9 & Azahar & 121904 & III & 29 & 23 & 22 \\
\hline A10 & Azahar & 151429 & III & 26 & 20 & 18 \\
\hline A11 & Azahar & 75650 & II & 32 & 26 & 20 \\
\hline E1 & Eucalipto & 306000 & III & 19 & 16 & 14 \\
\hline E2 & Eucalipto & 322000 & III & 24 & 21 & 15 \\
\hline E3 & Eucalipto & 248100 & III & 19 & 16 & 12 \\
\hline E4 & Eucalipto & 427917 & III & 20 & 16 & 13 \\
\hline E5 & Eucalipto & 348000 & III & 24 & 20 & 16 \\
\hline E6 & Eucalipto & 319000 & III & 23 & 19 & 14 \\
\hline E7 & Eucalipto & 285454 & III & 20 & 16 & 14 \\
\hline E8 & Eucalipto & 301500 & III & 24 & 19 & 18 \\
\hline E9 & Eucalipto & 556500 & IV & 22 & 18 & 11 \\
\hline E10 & Eucalipto & 430500 & III & 34 & 25 & 23 \\
\hline R1 & Romero & 70000 & II & 37 & 31 & 24 \\
\hline $\mathrm{R} 2$ & Romero & 87500 & II & 36 & 29 & 25 \\
\hline $\mathrm{R} 3$ & Romero & 58000 & II & 37 & 30 & 26 \\
\hline $\mathrm{R} 4$ & Romero & 68500 & II & 33 & 27 & 21 \\
\hline $\mathrm{R} 5$ & Romero & 82000 & II & 35 & 29 & 23 \\
\hline R6 & Romero & 111429 & III & 37 & 29 & 28 \\
\hline $\mathrm{R} 7$ & Romero & 59505 & II & 38 & 32 & 23 \\
\hline
\end{tabular}


Tabla 2

Espectro polínico medio de las mieles analizadas según su tipo comercial. * Espectro polínico medio completo. ** Espectro polínico medio eliminadas las especies poliníferas

\begin{tabular}{|c|c|c|c|c|c|c|}
\hline \multirow{2}{*}{ Formas polínicas } & \multicolumn{2}{|c|}{ Romero } & \multicolumn{2}{|c|}{ Azahar } & \multicolumn{2}{|c|}{ Eucalipto } \\
\hline & $*$ & $* *$ & $*$ & $* *$ & $*$ & $* *$ \\
\hline ACANTHACEAE & & & & & & \\
\hline $\begin{array}{l}\text { Stenandrium sp. } \\
\text { ACERACEAE }\end{array}$ & & & $0,10 \%$ & $0,17 \%$ & & \\
\hline $\begin{array}{l}\text { Acer sp. } \\
\text { BORAGINACEAE }\end{array}$ & $0,45 \%$ & $0,53 \%$ & $0,76 \%$ & $1,08 \%$ & & \\
\hline Anchusa azurea & $0,19 \%$ & $0,22 \%$ & & & & \\
\hline Echium plantagineum & $7,68 \%$ & $9,98 \%$ & $13,20 \%$ & $20,38 \%$ & $10,24 \%$ & $11,09 \%$ \\
\hline $\begin{array}{l}\text { Myosotis sp. } \\
\text { CAESALPINACEAE }\end{array}$ & $0,03 \%$ & $0,04 \%$ & & & & \\
\hline $\begin{array}{l}\text { Enterolobium sp. } \\
\text { CAMPANULACEAE }\end{array}$ & & & & & $0,01 \%$ & $0,01 \%$ \\
\hline $\begin{array}{l}\text { Campanula erinus } \\
\text { CARYOPHYLLACEAE }\end{array}$ & $1,20 \%$ & $1,50 \%$ & $0,66 \%$ & $1,11 \%$ & $0,83 \%$ & $0,87 \%$ \\
\hline $\begin{array}{l}\text { Silene vulgaris } \\
\text { CAESALPINIACEAE }\end{array}$ & $0,40 \%$ & $0,51 \%$ & & & & \\
\hline $\begin{array}{l}\text { Ceratonia siliqua } \\
\text { CISTACEAE }\end{array}$ & $0,42 \%$ & $0,49 \%$ & & & & \\
\hline Cistus ladanifer & $3,11 \%$ & & $3,41 \%$ & & $3,61 \%$ & \\
\hline $\begin{array}{l}\text { Otras Cistaceae } \\
\text { COMPOSITAE }\end{array}$ & $16,28 \%$ & & $18,08 \%$ & & $1,30 \%$ & \\
\hline Anthemis arvensis & $0,30 \%$ & $0,37 \%$ & & & & \\
\hline Calendula arvensis & $1,26 \%$ & $1,58 \%$ & $0,11 \%$ & $0,19 \%$ & $0,21 \%$ & $0,22 \%$ \\
\hline Centaurea calcitrapa & $0,03 \%$ & $0,04 \%$ & $0,01 \%$ & $0,05 \%$ & & \\
\hline Crepis capillaris & $0,13 \%$ & $0,15 \%$ & $0,03 \%$ & $0,09 \%$ & & \\
\hline Liguliflorae & $0,35 \%$ & $0,43 \%$ & $0,64 \%$ & $0,71 \%$ & $0,02 \%$ & $0,02 \%$ \\
\hline $\begin{array}{l}\text { Senecio vulgaris } \\
\text { CONVOLVULACEAE }\end{array}$ & $0,06 \%$ & $0,08 \%$ & & & $0,17 \%$ & $0,18 \%$ \\
\hline $\begin{array}{l}\text { Convolvulus arvensis } \\
\text { CRUCIFERAE }\end{array}$ & $0,17 \%$ & $0,27 \%$ & & & & \\
\hline Rhapanus raphanistrum & $3,65 \%$ & $4,88 \%$ & $2,77 \%$ & $4,55 \%$ & $0,37 \%$ & $0,39 \%$ \\
\hline $\begin{array}{l}\text { Sinapis arvensis } \\
\text { CHENOPODIACEAE }\end{array}$ & $3,35 \%$ & $4,04 \%$ & $0,90 \%$ & $2,25 \%$ & & \\
\hline $\begin{array}{l}\text { Chenopodium album } \\
\text { ERICACEAE }\end{array}$ & $0,28 \%$ & & & & $0,01 \%$ & \\
\hline Erica arborea & $1,36 \%$ & $1,80 \%$ & $0,00 \%$ & $0,01 \%$ & $0,24 \%$ & $0,26 \%$ \\
\hline $\begin{array}{l}\text { Erica australis } \\
\text { EUPHORBIACEAE }\end{array}$ & $0,28 \%$ & $0,41 \%$ & $0,00 \%$ & $0,01 \%$ & $0,30 \%$ & $0,31 \%$ \\
\hline $\begin{array}{l}\text { Euphorbia helioscopica } \\
\text { FAGACEAE }\end{array}$ & $0,35 \%$ & $0,43 \%$ & & & & \\
\hline Castanea sativa & & & & & $0,66 \%$ & $0,71 \%$ \\
\hline $\begin{array}{l}\text { Quercus coccifera } \\
\text { FUMARIACEAE }\end{array}$ & $1,50 \%$ & & $3,11 \%$ & & $0,15 \%$ & \\
\hline Hypecoum imberbe & $1,95 \%$ & $2,76 \%$ & $0,75 \%$ & $1,15 \%$ & $0,22 \%$ & $0,24 \%$ \\
\hline
\end{tabular}




\begin{tabular}{|c|c|c|c|c|c|c|}
\hline \multirow{2}{*}{ Formas polínicas } & \multicolumn{2}{|c|}{ Romero } & \multicolumn{2}{|c|}{ Azahar } & \multicolumn{2}{|c|}{ Eucalipto } \\
\hline & * & $* *$ & $*$ & $* *$ & * & ** \\
\hline \multicolumn{7}{|l|}{ GRAMINAE } \\
\hline Festuca arundinacea & $0,46 \%$ & & $0,75 \%$ & & $0,08 \%$ & \\
\hline Zea mays & $0,13 \%$ & & & & $0,08 \%$ & \\
\hline \multicolumn{7}{|l|}{ GROSSULARIACEAE } \\
\hline Ribes sp. & $0,09 \%$ & $0,16 \%$ & & & & \\
\hline \multicolumn{7}{|l|}{ HALORAGACEAE } \\
\hline \multicolumn{6}{|l|}{ LABIATAE } & $0,01 \%$ \\
\hline Lamium amplexicaule & $0,22 \%$ & $0,29 \%$ & & & & \\
\hline Lavandula stoechas & $3,75 \%$ & $5,13 \%$ & $0,58 \%$ & $0,88 \%$ & $0,43 \%$ & $0,46 \%$ \\
\hline Mentha aquatica & $1,28 \%$ & $1,72 \%$ & $0,04 \%$ & $0,01 \%$ & $0,03 \%$ & $0,03 \%$ \\
\hline Salvia verbenaca & $13,05 \%$ & $17,23 \%$ & $0,53 \%$ & $0,90 \%$ & $0,28 \%$ & $0,30 \%$ \\
\hline \multicolumn{7}{|l|}{ LEGUMINOSAE } \\
\hline Anthyllis sp. & $2,56 \%$ & $3,65 \%$ & & & & \\
\hline Cytisus scoparius & $7,43 \%$ & $10,75 \%$ & $5,62 \%$ & $7,22 \%$ & $1,20 \%$ & $1,30 \%$ \\
\hline Lotus creticus & $0,91 \%$ & $1,29 \%$ & $1,59 \%$ & $2,78 \%$ & $0,43 \%$ & $0,46 \%$ \\
\hline Onobrychis viciifola & $0,45 \%$ & $0,55 \%$ & & & & \\
\hline Psoralea bituminosa & & & $0,01 \%$ & $0,03 \%$ & & \\
\hline Spartium junceum & $0,02 \%$ & $0,03 \%$ & & & & \\
\hline Trifolium arvense & $0,81 \%$ & $1,17 \%$ & $1,20 \%$ & $1,73 \%$ & $0,24 \%$ & $0,25 \%$ \\
\hline Trifolium pratense & $0,06 \%$ & $0,08 \%$ & & & $0,01 \%$ & $0,01 \%$ \\
\hline Trifolium repens & $0,53 \%$ & $0,77 \%$ & $0,37 \%$ & $0,74 \%$ & $0,35 \%$ & $0,38 \%$ \\
\hline \multicolumn{7}{|l|}{ LILIACEAE } \\
\hline Allium sp. & & & & & $0,09 \%$ & $0,09 \%$ \\
\hline Asphodelus fistulosus & $0,26 \%$ & $0,29 \%$ & & & & \\
\hline Lilium $\mathrm{sp}$ & $0,78 \%$ & $0,99 \%$ & $0,58 \%$ & $0,74 \%$ & $0,34 \%$ & $0,36 \%$ \\
\hline Otras Liliaceae & $0,15 \%$ & $0,17 \%$ & & & & \\
\hline \multicolumn{7}{|l|}{ LYTHRACEAE } \\
\hline Lytrhum salicaria & $0,33 \%$ & $0,49 \%$ & $0,00 \%$ & $0,00 \%$ & $0,45 \%$ & $0,58 \%$ \\
\hline MYRTACEAE & & & & & & \\
\hline Myrtus communis & $4,09 \%$ & $5,66 \%$ & $7,05 \%$ & $11,97 \%$ & $73,99 \%$ & $78,32 \%$ \\
\hline \multicolumn{7}{|l|}{ OLEACEAE } \\
\hline Ligustrum vulgare & $0,80 \%$ & $0,95 \%$ & $0,62 \%$ & $1,07 \%$ & & \\
\hline Olea europea & $0,84 \%$ & & $2,55 \%$ & & $0,02 \%$ & \\
\hline \multicolumn{7}{|l|}{ ONAGRACEAE } \\
\hline Epilobium parviflorum & $0,20 \%$ & $0,26 \%$ & & & & \\
\hline \multicolumn{7}{|l|}{ OXALIDACEAE } \\
\hline Oxalis sp. & $0,32 \%$ & $0,45 \%$ & $1,06 \%$ & $1,59 \%$ & & \\
\hline \multicolumn{7}{|l|}{ PAPAVERACEAE } \\
\hline Papaver rhoeas & $0,38 \%$ & & & & & \\
\hline \multicolumn{7}{|l|}{ PLANTAGINACEAE } \\
\hline Plantago coronopus & $1,17 \%$ & & $2,43 \%$ & & $0,28 \%$ & \\
\hline POLYGALACEAE & & & & & & \\
\hline Polygala sp. & $0,06 \%$ & $0,08 \%$ & & & & \\
\hline POLYGONACEAE & & & & & & \\
\hline Polygonum sp. & $0,06 \%$ & $0,08 \%$ & & & & \\
\hline
\end{tabular}




\begin{tabular}{|c|c|c|c|c|c|c|}
\hline \multirow{2}{*}{ Formas polínicas } & \multicolumn{2}{|c|}{ Romero } & \multicolumn{2}{|c|}{ Azahar } & \multicolumn{2}{|c|}{ Eucalipto } \\
\hline & $*$ & $* *$ & $*$ & $* *$ & $*$ & $* *$ \\
\hline \multicolumn{7}{|l|}{ RHAMNACEAE } \\
\hline $\begin{array}{l}\text { Rhamnus alaternus } \\
\text { ROSACEAE }\end{array}$ & $0,03 \%$ & $0,04 \%$ & $0,36 \%$ & $0,47 \%$ & & \\
\hline Crataegus monogyna & $3,81 \%$ & $4,62 \%$ & $3,17 \%$ & $4,86 \%$ & $0,10 \%$ & $0,12 \%$ \\
\hline Gerum sp. & & & $0,24 \%$ & $0,23 \%$ & & \\
\hline Prunus spinosa & $1,85 \%$ & $2,47 \%$ & $2,35 \%$ & $3,37 \%$ & $0,52 \%$ & $0,55 \%$ \\
\hline Rubus ulmifolius & $1,39 \%$ & $1,89 \%$ & $1,69 \%$ & $2,24 \%$ & $0,36 \%$ & $0,39 \%$ \\
\hline \multicolumn{7}{|l|}{ RANUNCULACEAE } \\
\hline Thalictrum speciosissimum & $0,40 \%$ & $0,56 \%$ & & & & \\
\hline RHAMNACEAE & & $0,00 \%$ & & & & \\
\hline Rhamnus alaternus & $0,83 \%$ & $0,94 \%$ & & & & \\
\hline RUBIACEAE & & $0,00 \%$ & & & & \\
\hline Galium aparine & $0,16 \%$ & $0,20 \%$ & & & & \\
\hline RUTACEAE & & $0,00 \%$ & & & & \\
\hline Citrus sinensis & $1,76 \%$ & $2,24 \%$ & $14,29 \%$ & $17,74 \%$ & $0,11 \%$ & $0,12 \%$ \\
\hline SALICACEAE & & $0,00 \%$ & & & & \\
\hline Salix sp. & $1,15 \%$ & $1,48 \%$ & $1,57 \%$ & $2,49 \%$ & $0,01 \%$ & $0,01 \%$ \\
\hline SCROPHULARIACEAE & & $0,00 \%$ & & & & \\
\hline Bellardia trixago & $0,25 \%$ & $0,35 \%$ & & & & \\
\hline Pedicularis sylvatica & $0,24 \%$ & $0,38 \%$ & & & & \\
\hline SOLANACEAE & & $0,00 \%$ & & & & \\
\hline Solanum nigrum & $0,25 \%$ & $0,32 \%$ & $0,01 \%$ & $0,05 \%$ & $0,01 \%$ & $0,01 \%$ \\
\hline TILIACEAE & & $0,00 \%$ & & & & \\
\hline Tilia platyphyllos & $0,23 \%$ & $0,27 \%$ & & & & \\
\hline UMBELLIFERAE & & $0,00 \%$ & & & & \\
\hline Conium maculatum & & & $0,23 \%$ & $0,42 \%$ & $0,10 \%$ & $0,10 \%$ \\
\hline Daucus carota & $0,06 \%$ & $0,08 \%$ & $0,01 \%$ & $0,03 \%$ & $0,01 \%$ & $0,02 \%$ \\
\hline VITACEAE & & $0,00 \%$ & & & & \\
\hline Vitis vinifera & $0,56 \%$ & $0,74 \%$ & $1,10 \%$ & $1,85 \%$ & $0,04 \%$ & $0,06 \%$ \\
\hline OTROS & $0,71 \%$ & $0,86 \%$ & $2,91 \%$ & $3,94 \%$ & $1,60 \%$ & $1,90 \%$ \\
\hline
\end{tabular}

estuvieron presentes en todas las muestras estudiadas. De igual forma se repitieron un total de trece formas polínicas a lo largo de todas las mieles inscritas en este grupo, con porcentajes variables en función de la muestra tratada. El tipo polínico Cistus ladanifer, junto con la forma polínica en la que agrupamos al resto de cistáceas presentes en todas las muestras alcanzaron porcentajes de representación muy elevados en algunos casos, como en A9 donde, en conjunto, la familia de las cistáceas ocupó más del 50\% del polen identificado. Los tipos Quercus coccifera, Olea europaea y Plantago coronopus, también poliníferos, no superaron porcentajes del 10\% en ninguna de las mieles de azahar analizadas, aunque cabe reseñar el 9,3\% que alcanzó el primer tipo en A3. El resto de tipos representados en todas las muestras son tipos adscritos a especies que aportan néctar a la miel, destacando los tipos Citrus sinensis, Echium plantagineum y Myrtus communis que presentaron porcentajes superiores al $20 \%$ en algunas muestras (A1, A2 y A11 en el primero de los tipos, A1, A5, A8, A9 y A10 en el segundo, y A3 en el último) y mostraron un porcentaje medio de representación de $20 \%$ (para los dos primeros) y $11 \%$, respectivamente (Fig. 1). Los tipos Cytisus scoparius, Raphanus raphanistrum y Prunus spinosa, con porcentajes medios de $8 \%, 4 \%$ y $3 \%$, no superaron el $10 \%$ en ninguna de las mieles analizadas, salvo el tipo que representa a retamas y escobas en las muestras A4, A5, A6 y A10. También estuvieron representados los tipos Lotus creticus y Sinapis arvensis, no 
superando el $4 \%$ en ninguna de las muestras ni rebasando porcentajes medios del 3\%. En menor medida, hemos de destacar la presencia del tipo Vitis vinifera y de la forma polínica Lilium sp. en diez de las mieles etiquetadas como "azahar", así como de los tipos Campanula erinus, Crataegus monogyna y la forma Liguliflorae en ocho. El tipo polínico Trifolium arvense, presente en siete muestras, obtuvo un $6,8 \%$ de representación en A6, mientras que la forma polínica Acer sp., sólo presente en seis, alcanzó un $8,1 \%$ en A1, y el tipo Stenandrium dulce, una forma ajena a la flora peninsular, apareció en A4 $(1,1 \%)$.

Un total de 42 formas polínicas pertenecientes a 26 familias botánicas fueron identificadas en las diez mieles de eucalipto adquiridas en el período 2002-2008, con una media de 23 formas polínicas incluidas en 19 familias. Dentro de estas últimas, la familia Leguminosae con 5 tipos polínicos, es la que presentó mayor diversidad polínica, seguida por las familias Compositae y Labiatae, con 3. Las mieles de eucalipto nominadas como E8 y E7 son las que mostraron la mayor y la menor riqueza polínica, respectivamente. En las diez muestras estudiadas se repitieron un total de once formas polínicas cuyos porcentajes de representación variaron en función de la muestra analizada. Dentro de las formas nectaríferas, destaca Myrtus communis, que con un porcentaje medio de representación del 78 alcanza el 90\% en dos muestras (E2 y E3), y Echium plantagineum, que superó el 20\% en la muestra E5 y presentó un porcentaje medio del 11\% (Fig. 1). El resto de formas que aportan néctar a la miel localizadas en todas las muestras (Campanula erinus, Cytisus scoparius, Lotus creticus, Prunus spinosa, Senecio vulgaris) presentaron porcentajes medios bajos, salvo los tipos polínicos Cytisus scoparius con $1 \%$ de representación media y un 3,4\% en E8, y Campanula erinus, que alcanza un 3,5\% en E7. Dos tipos polínicos pertenecientes a la familia Labiatae, Lavandula stoechas y Salvia verbe$n a c a$, aparecieron en 9 de las muestras analizadas pero también con porcentajes muy bajos. Las familias poliníferas presentes en todas las muestras de miel de eucalipto son Cistaceae y Plantaginaceae no presentaron porcentajes de representación importantes dentro de las formas polínicas que incluyen, salvo el tipo Cistus ladanifer que superó el 17\% en la muestra E10. En la muestra E2 se identificó un grano de polen del género Enterolobium, propio del continente americano.

Las siete muestras de mieles de romero estudiadas presentaron 65 formas polínicas en total adscritas a 40 familias botánicas, y una media de 36 formas incluidas dentro de 29 familias. La familia Leguminosae es la que obtuvo una diversidad polínica mayor, ya que en ella se adscribie- ron ocho de las formas polínicas identificadas, seguidas por Compositae, con 6, y Labiatae, con 4. En lo que respecta a la riqueza polínica de las muestras estudiadas, debe señalarse a R6 y R4, como aquellas que presentaron mayor y menor riqueza, respectivamente. Dentro del espectro polínico obtenido en las mieles de romero aparecieron 16 formas en todas las muestras, destacando el tipo polínico Cistus ladanifer y la forma "Otras Cistaceae", que en conjunto supusieron más de un tercio del polen identificado en R3 y R5. El otro tipo polínico que engloba a especies poliníferas presente en todas las muestras, Plantago coronopus, dio lugar a porcentajes medios de representación bajos, no rebasando el 3,5\% en ninguna de las muestras. El resto de formas polínicas identificadas en todas las muestras incluyen a especies botánicas que aportan néctar a la miel, destacando el tipo polínico Salvia verbenaca, con un porcentaje medio de representación del $17 \%$ y superando el 20\% en R6. Otros tipos presentes en todas las mieles como Crataegus monogyna, Cytisus scoparius, Echium plantagineum y Myrtus communis, superaron el $10 \%$ en varias de las muestras (Fig. 1), aunque con porcentajes medios de representación diferentes, $5 \%$, $11 \%, 10 \%$, y $5 \%$, respectivamente. Además, los tipos $L a$ vandula stoechas, Crataegus monogyna e Hypecoum imberbe, que rebasaron el 2\% de representación media, Erica arborea, Lotus creticus, Prunus spinosa, Rubus ulmifolius y las formas Lilium sp. y Salix sp., también están presentes en todas las muestras y con porcentajes de representación medios inferiores al $2 \%$.

\section{DISCUSIÓN}

El número de elementos botánicos presentes en el sedimento de todas las mieles analizadas, incluidos en las Clases II y III, salvo una muestra de miel de eucalipto (E9) englobada dentro de la Clase IV, parece indicar que todas estas mieles fueron obtenidas tras procesos de centrifugado, aunque dicha información no se especifica en el etiquetado del producto. Sin embargo, la alta representación de la familia Cistaceae en el espectro polínico de las mieles de azahar y romero podría suponer que dichas mieles fueran obtenidas por procesos de prensado o bien como una contaminación en el interior de las colmenas (Loveaux 1958, Ortiz 1990). No obstante, y aunque algunos autores hablan de la producción de néctar por parte de especies de esta familia (Herrera 1985, Talavera et al. 1988), no existen estudios que revelen el grado de representatividad de estos granos de polen y su contribución dentro de las mieles de flores, por lo que estimamos que esta al- 
ta incidencia fue debida a una contaminación dentro de la colmena, gracias a la elevada presencia de esta familia en el polen apícola peninsular (Cordón Marcos 2005). Por otro lado, el número de ECM, nulo o casi nulo en todas las muestras estudiadas, salvo dos muestras de azahar y una de eucalipto con índices de mielada muy bajos, indica que todas estas mieles eran mieles de néctar.

Las once mieles que recibieron la denominación de azahar por parte de la empresa comercial presentaron porcentajes de representación diversos para el tipo polínico Citrus sinensis, que engloba a diversas especies de árboles frutales de la familia de las rutáceas, entre los cuales destacan el naranjo y el limonero. Dichos porcentajes son considerados sólo a través de especies nectaríferas y, por tanto, deben eliminarse del espectro a todas aquellas formas polínicas que no aporten néctar a la miel. Como consecuencia de la ya comentada falta de legislación al respecto del porcentaje que una determinada forma polínica ha de poseer para considerar a una miel como monofloral de dicho tipo, Serra Bonheví et al. (1987) concretan el $15 \%$ como valor adecuado para considerar una miel como monofloral de azahar, mientras que en otro trabajo del sureste peninsular (Munuera \& Carrión 1994) este porcentaje alcanza el 25\%. En este caso, y debido a los recientes cultivos de cítricos estériles, que dan lugar a flores productoras de néctar pero no de polen, consideramos válido el porcentaje del $10 \%$ para delimitar la monofloralidad de estas muestras (Molins Marín et al. 1995). Por lo tanto, consideramos que desde el punto de vista polínico la marca comercial cumple con los criterios botánicos necesarios, ya que todas las muestras salvo A5 con un 9,6\%, sobrepasan el mencionado porcentaje. La tipificación botánica de las mieles analizadas concuerda con los estudios realizados en el este de la Península Ibérica, con la presencia de los tipos polínicos Echium plantagineum, Myrtus communis, Olea europaea, Plantago coronopus, Quercus coccifera y Salvia verbenaca, junto a diversos representantes de las familias Cruciferae, Cistaceae, Compositae, Leguminosae o Vitaceae.

No obstante, hemos de significar la aparición de la familia Rosaceae, con los tipos Crataegus monogyna y Prunus spinosa, y del tipo Campanula erinus, importantes en las muestras apícolas de España y Portugal, como caracteres diferenciales más destacados con respecto a otras mieles de este origen floral. En todos los casos, podríamos certificar que las mieles de azahar tienen un origen enteramente español, tal y como se indica en la etiqueta del producto, pese a que en la muestra A4 hayamos detectado la presencia, poco relevante desde un punto de vista cuantitativo, de varios granos de polen adscritos al tipo polínico Stenandrium dulce, propio de una especie herbácea distribuida por todo el continente americano. Dado que la mitad de la miel importada a la Unión Europea procedió de Argentina (Tercer Informe sobre la aplicación del Reglamento (CE) nº 797/2004), la miel nominada como A4 podría haber sido mezclada con una pequeña cantidad de alguna miel de néctar argentina que tuviera caracteres organolépticos similares a los que presentan las mieles de azahar de origen español.

Las mieles de eucalipto han sido muy estudiadas en numerosos trabajos y con diferentes umbrales de representatividad del tipo polínico Myrtus communis, ya que en algunos casos se establece en un 70\% (Loveaux et al. 1978, Serra 1989, Sala-Llinares 1991) y en otros en un $90 \%$ (Maurizio 1979). En nuestro caso, y dado que la legislación argentina sí exige un porcentaje mayor al 70\% para este tipo de mieles en (Resolución 1051/94 sobre tipificación de las mieles por el origen botánico), consideramos como válido un porcentaje de, al menos, el $80 \%$ del total de elementos botánicos procedentes de especies nectaríferas que nos permita diferenciar mieles peninsulares de eucalipto de las argentinas. Atendiendo a este criterio, sólo la mitad de las muestras serían consideradas como monoflorales, las correspondientes a los años 2002 y 2003, más la muestra de 2006. Respecto a la caracterización botánica, hemos de señalar la presencia de los tipos polínicos Castanea sativa, Rubus ulmifolius, Quercus coccifera y la familia de las ericáceas, ya que son formas polínicas que indican el origen peninsular de estas mieles (Seijo et al. 2003) con respecto a las procedentes de Australia. En este mismo sentido, la aparición de los tipos polínicos Olea europaea, Hypecoum imberbe y la familia de las cistáceas, nos permite descartar el origen argentino de las muestras (Sala-Llinares \& Suárez-Cervera, 1985). Dentro de este tipo de mieles producidas en la Península Ibérica, la ausencia de las formas Heracleum, Reseda y Scrophularia en las mieles comerciales estudiadas (Seijo et al. 2003), así como la presencia de los tipos Cistus ladanifer, Citrus sinensis, Hypecoum imberbe, Lavandula stoechas y Olea europaea (Seijo et al., 1999) nos indican que estas mieles no fueron producidas en Portugal y Galicia. Junto a estas formas polínicas, debe destacarse además la presencia del tipo Campanula erinus en todas las muestras y que podría indicarnos el lugar de origen de estas mieles, pues se trata de un tipo polínico muy habitual en mieles del centrooeste español (Barrios Pérez \& Sánchez Sánchez 2003), y del tipo Enterolobium contortisiliqum en la muestra E2, que indicaría, como en el caso de la muestra A4, que esta partida comercial podría estar constituida por una mezcla de mieles, entre las que se encontraría una miel de origen 
sudamericano, preferentemente argentino, debido al volumen de exportaciones que posee este país. En todo caso, y salvo la particularidad ya comentada, todas las mieles presentaron tipos polínicos propios de la flora peninsular, delimitando así su origen geográfico.

La representatividad del tipo polínico Salvia verbe$n a c a$, que incluye a varias especies de labiadas, entre ellas el romero (Rosmarinus officinalis L.), ha sido objeto de estudio por parte de varios autores. Lavie (1976) considera suficiente un $10 \%$ para considerar una miel como monofloral de romero, mientras que otros autores (Serra Bonheví et al. 1987, Gómez Ferreras 1988) fijan entre el $15-20 \%$ de dominancia para determinar su monofloralidad, porcentaje que estimamos oportuno debido a la hiporrepresentación de este tipo en las mieles y a la ausencia de los fenómenos de esterilización forzada que señalamos en el caso de la miel de azahar. Este porcentaje es considerado sólo a través de especies nectaríferas y, por tanto, debe eliminarse del espectro a todas las formas polínicas que incluyan especies que no aporten néctar a la miel. Así pues, todas las muestras analizadas, salvo R2, sobrepasan este umbral, por lo que desde el punto de vista botánico y palinológico, las mieles de romero puestas a la venta por la misma casa comercial, cumplen los criterios recomendados.

Además, estas mieles también cumplen los requerimientos geográficos por poseer un espectro con formas polínicas que engloban a especies propias de la Península Ibérica, y por la presencia de los tipos Hypecoum imberbe, Prunus spinosa, Cistus ladanifer, Cytisus scoparius, y Mentha aquatica señalados por otros autores ya referidos. No obstante, conviene reseñar la presencia de los tipos Crataegus monogyna, Lavandula stoechas y Rubus ulmifolius, como elementos claramente diferenciadores de las mieles estudiadas, junto con los géneros Acer y Lilium, con respecto a las analizadas en los trabajos referidos.

\section{BIBLIOGRAFÍA}

Barrios Pérez, J. \& SÁnchez SÁnchez, J. 2003 . Análisis polínico de mieles de "bosque"del centro-oeste español (Cáceres y Salamanca). Polen 13: 333-339.

Carretero, J. L. 1989. Análisis polinico de la miel. Ed. MundiPrensa, Madrid.

Cordón Marcos, C. 2005. Palinología y caracteres físicoquímicos del polen apicola producido en España. Propuesta de parámetros objetivos de calidad. Tesis Doctoral, Universidad de Salamanca, Salamanca.

De Sá Otero, P.; Armesto Baztán, S. \& Díaz Losada, E. 2006. A study of variation in the pollen spectra of honeys sampled from Baixa-Limia Serra do Xurés Nature Reserve in northwest of Spain. Grana 45: 137-145.

de Sá Otero, P.; Díaz Losada, E. \& Armesto Baztán, S. 2011. Caracterización de mieles de obtención artesanal y comerciales producidas en Galicia (NO de España) a partir de su espectro polínico y contenido proteico. Bot. Complut. 35: 131-140.

ERDTMAN, G. 1960. The acetolysis method. A revised description. Svenk. Bot. Tidskr. 54: 561-564.

Gómez Ferreras, C. 1988. Análisis polínico de mieles de la provincia de Madrid (España). En: J. Civis Llovera \& M. Valle Hernández (Eds.), Actas del VI Simposio de Palinologia, APLE: 223-230. Universidad de Salamanca, Salamanca.

HERRERA, J. 1985. Nectar secretion patterns in southern Spanish Mediterranean shrublands. Israel J. Bot. 34: 47-58.

Heusser, C. J. 1971. Pollen and spores of Chile. Modern types of the Pteridophyta, Gymnospermae and Angiospermae. The University of Arizona Press. Tucson, Arizona.

HidEUX, M. 1972. Techniques d'étude du pollen an MED: effects comparés des différents traitements physicochimiques. Micron 3: 1-31.
Jones, G. D. \& Bryant, V. M. 1996. Melissopalynology. En: J. Jansonius \& D. C. McGregor (Eds.), Palynology: principles and applications: 933-938. American Association of Statigraphic Palynologists Foundation, Texas.

LAVIE, P. 1976. Les plantes mellifëres. Le romarin: Rosmarinus officinalis. L.B.T.A. 1: 15-26.

LOVEAUX, J. 1958. Recherches sur l'origine dans le miel du pollen de plantes entomophiles depourvues de nectaires. Ann. Abeille 1(2): 89-92.

LoveauX, J.; Maurizio, A. \& Vorwhol, G. 1978. Methods of melissopalynology. Bee World 59(4): 139-157.

Markgraf, V. \& D'Antoni, H. L. 1978. Pollen flora of Argentina. Modern spore and pollen types of Pteridophyta, Gymnospermae and Angiospermae. The University of Arizona Press. Tucson, Arizona.

MAuRIZIo, A. 1939. Untersuchungen zur quantitativen pollenlyse des honing. Mitt. Geb. Lebensmittelunters 30: 27-69.

Maurizio, A. 1979. Microscopy of honey. En: E. Crane (Ed.), Honey. A comprehensive survey: 240-257. Heinemann, London.

Molins Marín, J. L.; Perea López, F.; Montilla Gómez, J.; MarTínez Gómez, E. \& GuerRa Hernández, E. 1995. Caractérisation du miel d'oranger (Citrus sp.) produit en Espagne, au moyen de son spectre pollinique. Lagascalia 18(1): 71-82.

Munuera Giner, M. \& CARRión García, J. S. 1994. Análisis polínico de mieles de azahar de la Vega del Segura (Alicante y Murcia). Aliment. 258: 37-42.

OrTIZ, P. L. 1990. Contribución al conocimiento de la flora apícola gaditana. Lagascalia 16(2): 199-210.

PéreZ De ZaBALZA, A. I. 1989. Estudio palinológico de mieles de Navarra. Tesis Doctoral, Universidad de Navarra, Pamplona. 
Sala-Luinares, A. 1991. Estudi palinològic de les mels de la mediterrània occidental. Comparaciò amb mels d'altres origens. Tesis Doctoral, Universidad de Barcelona, Barcelona.

Sala-Llinares, A. \& Sú́rez-Cervera, M. 1985. Sobre la existencia de indicadores polínicos en mieles argentinas de importación. Polen 2: 361-368.

Sánchez Sánchez, J.; Gómez Barez, J. A.; Escribano González, M. C. \& García-Villanova Ruiz, R. J. 1996. Análisis de mieles comerciales de la provincia de Salamanca. Bot. Macaronésica 23: 167-175.

Seijo Coello, M. C. \& Aira Rodríguez, M. J. 1999. Tipos polínicos de las miles comercializadas de Galicia. Acta Bot. Malac. 24: 101-111.

Seijo, M. C.; Aira, M. J. \& MÉndez, J. 2003. Palynological differences in the pollen content of Eucalyptus honey from Australia, Portugal and Spain. Grana 42: 183-190.

SerRA, J. 1989. Características físico-químicas. Composición de la miel de eucalipto (Eucalyptus sp.) producida en España. An. Bromatol. 41: 41-56.
Serra Bonheví, J.; Gómez Pajuelo, A. \& Gonell, J. 1987. Composición, propiedades físico-químicas y espectro polínico de algunas mieles monoflorales de España. Aliment. 185: 61-84.

Talavera, S.; Herrera, J.; Arroyo, J.; Ortiz, P. L. \& Devesa, J. A. 1988. Estudio de la flora apícola de Andalucía occidental. Lagascalia 15 (extra): 567-591.

TerRab, A.; ANDrés, C. \& DízZ, M. J. 2004a. Análisis polínico de mieles en el Parque Natural Sierra de Aracena y Picos de Aroche. Bot. Complut. 28: 121-126.

Terrab, A.; Pontes, A.; Heredia, F. J. \& Díez, M. J. 2004b. Palynological and geographical characterization of avocado honeys in Spain. Grana 43: 116-121.

Valdés, B.; Díez, M. J. \& Fernández, I. 1987. Atlas polínico de Andalucía occidental. Instituto de Desarrollo Regional y Excma. Diputación de Cádiz, Sevilla. 